\title{
Loudness and reaction time: II Identification of detection components at different intensities and frequencies
}

\author{
DAVID L. KOHFELD, JEFFREY L. SANTEE, and NORVAL D. WALLACE \\ Southern Illinois University, Edwardsville, Illinois 62026
}

\begin{abstract}
Equal-loudness contours were first obtained for five stimulus frequencies at four stimulus intensities. These 20 stimuli were then presented as reaction-time signals in a Donders $\mathrm{C}$ paradigm. The Z-transform method of convolution, as applied in linear systems identification, was used to deconvolve an empirically generated response (or "residual") distribution ( $\mathrm{T}_{\mathrm{R}}$ ) from each of the 20 reaction-time (RT) distributions obtained at different intensities and frequencies. The resulting sensory-detection $\left(t_{d}\right)$ models formed exponential densities at strong intensities (60 and 80 phons), but their shapes were either gamma or normal at relatively weak intensities (20 and 40 phons). Our analyses support the idea that the simple reactiontime process (RT) is a convolution (or sum) of two component stages: stimulus detection ( $t_{d}$ ), followed by response evocation $\left(t_{r}\right)$. Based on the shapes of $t_{d}$, a neural-impulse theory is offered to account for the detection of simple auditory RT signals.
\end{abstract}

In two previous papers (Kohfeld, Santee, \& Wallace, 1981; Santee \& Kohfeld, 1977), we reported that equally loud stimuli across five frequencies produced equal reaction times at 80 - and 60 -phon intensities, but at the 40 and 20-phon levels the latencies were longer at $1,000 \mathrm{~Hz}$ than at the higher and lower stimulus frequencies. In order to provide a rigorous evaluation of these results, our present intent is to analyze the observed RT distributions generated from 100-, 500-, $1,000-, 5,000-$, and 10,000-Hz signals at intensities of $20,40,60$, and 80 phons. Any dissimilarities among the shapes of these RT distributions, especially at the 20- and 40-phon levels, should provide some insights as to whether the components of the RT process are different across stimulus frequencies when equivalently weak signals are employed.

One basic premise in this paper is that signal detection and response initiation are the two component stages in the simple reaction time (RT) process. This notion is not new, as Green and Luce (1971), Hohle (1965), and McGill (1963) have proposed models of

This research was supported by Research Grant 93614, awarded to D. L. Kohfeld and N. D. Wallace by the Office of Research and Projects in the Graduate School at Southern Illinois University at Edwardsville. J. L. Santee is now at The Johns Hopkins University. We thank Edward C. Carterette, David M. Green, Bertram Scharf, and an anonymous reviewer for their editorial assistance. We are especially grateful to $R$. Duncan Luce for his review of our analytic methods. Robert Ruhl's management of our laboratory equipment is also acknowledged. A brief version of this paper was presented by D. L. Kohfeld at the annual meeting of The Psychonomic Society, Phoenix, Arizona, 1979. Requests for reprints should be sent to David L. Kohfeld, Department of Psychology, Southern Illinois University at Edwardsville, Edwardsville, Illinois 62026. the RT process which begin with the assumption that observed RT distributions represent a convolution of the densities of two component random variables, one of which reflects the detection, or decision process $\left(t_{d}\right)$, and the other having to do with the response or "residual" latency $\left(t_{r}\right)$. The forms of these component densities have been open to controversy; for example, McGill (1963) assumed that $t_{d}$ is normally distributed and $t_{r}$ is exponentially distributed, whereas Hohle (1965) argued for the exact opposite interpretation. More recently, Green and Luce (1971) attempted to find the shape of $t_{r}$ by constructing $t_{d}$ theoretically (based on a Poisson pulse model of sensory detection), and then deconvolving $t_{d}$ from the empirical $R T$ distribution. Although somewhat successful, Green and Luce were unable to identify a completely realistic model of $t_{r}$, thus leading them to conclude that their initial model of $t_{d}$ would require further revision.

In view of the difficulties encountered by Green and Luce in their attempt to derive a model of $t_{r}$, we decided to try two different approaches to the analysis of the RT components. First, instead of constructing a theoretical model of $t_{d}$ and then solving for $t_{r}$, we thought it might work to obtain an estimate of $t_{r}$ and subsequently to generate models of $t_{d}$. Essential!y, this is the same strategy employed by Green and Luce (1971), except that they started with a model of $t_{d}$, whereas we began with an estimate of $t_{r}$. Our rationale was based in part on Luce and Green's (1974) suggestion that the RT distribution that is generated from very strong signals should be identical to $t_{r}$, the residual distribution. This view reflects their belief that the time for sensory detection $\left(t_{d}\right)$ gets progressively shorter as signal intensity increases, until at 
some strong intensity level, the time required by $t_{d}$ in the overall RT process reaches zero. Accordingly, we reasoned that if a $110-\mathrm{dB}, \mathrm{SPL}$, tone served as the RT signal, then RT should approximate $t_{r}$, especially if a highly practiced subject were employed. This empirical estimate, $T_{R}$, of the response component, $t_{r}$, could then be deconvolved from other RT distributions at weaker intensities, across different frequencies, in order to generate a "family" of $t_{d}$ models.

The second major difference in our approach is the use of linear systems identification (Hsia, 1977; Sage \& Melsea, 1971) instead of Fourier analysis to identify and separate $t_{d}$ and $t_{r}$ from the RT distributions. Since this may be the first attempt to use systems identification in a RT study, we will devote an entire section in this paper to a description of this analytic procedure.

In summary, we believe that some degree of success in the identification of the component densities, $t_{d}$ and $t_{r}$, is necessary in order to accomplish our research goals. Specifically, we reasoned that reaction times to weak-intensity signals (e.g., 20 phons) involve longer detection times than RTs to strong-intensity signals (e.g., 80 phons). This would imply that the models of $t_{d}$ at 20 phons should have different shapes (larger means and standard deviations) than those derived at 80 phons. Moreover, the models of $t_{d}$ for 20-phon signals across stimulus frequencies should also differ, thus providing one way to account for the finding that equally loud stimuli do not yield the same RTs at weak intensity-levels. Correspondingly, the models of $t_{d}$ at 80 phons, across frequencies, are more likely to be similar, since at least the mean RTs are equal across the stimulus frequencies. In the main, we expected that signal detection $\left(t_{d}\right)$, an essential ingredient in the overall RT process, would take longer and be more variable for 20 - and 40 -phon signals, thus allowing for the effects of signal frequency on $t_{d}$ to be more apparent than when 60- and 80-phon signals were utilized.

\section{METHOD}

\section{Subject}

The subject in this study was J.S., the second author of this paper. J.S. was a 25 -year-old graduate student with a normal hearing threshold $\left(20 \mu \mathrm{N} / \mathrm{m}^{2}\right.$ at $\left.1,000 \mathrm{~Hz}\right)$. He was thoroughly practiced in both the equal-loudness matching techniques and the Donders Type $C$ reaction-time procedures that were required in this research. J.S. had already participated as a subject in our previous work (Kohfeld et al., 1981, Experiment 1), and his previous equalloudness data, along with his RT measures, provided the data for the present analysis.

\footnotetext{
Apparatus

A detailed description of the apparatus and procedures that were used for obtaining equal-loudness curves, and subsequent presentation of these signals in the RT paradigm, has been provided elsewhere (Kohfeld et al., 1981). In brief, during the loudnessmatching sessions, the subject was seated in a sound-attenuated chamber. The stimuli were generated by two audio oscillators and were presented monaurally through a set of calibrated earphones. Stimulus control consisted of appropriate signal attenuation, by
}

means of two decade attenuators, and switching between the standard and comparison tones. Specifically, the subject was presented with alternating bursts of a 1,000- $\mathrm{Hz}$ tone (left ear) at a standard intensity and the comparison tone (right ear) at a variable intensity, and was asked to adjust the comparison tone to match subjectively the $1,000-\mathrm{Hz}$ reference tone. An electronic switch was used to alternate the stimuli at 2.5-sec durations with rise and decay times of $10 \mathrm{msec}$. There was no interval between the standard and comparison tones. Two adjustable attenuators, controlled by the experimenter, were located outside the chamber. The attenuation of the comparison tone was adjusted by the experimenter based on the subject's verbal instructions (i.e., "up" or "down"). An intercom system provided means for subject-experimenter communications.

The RT sessions were also conducted while the subject was seated in the sound-attenuated chamber. Auditory signals were generated by an audio oscillator and, after appropriate attenuation, were presented to the subject binaurally through the same set of earphones that was used in the equal-loudness, matching sessions. The rise and decay times of the RT signals were $10 \mathrm{msec}$. On each trial, a 1-sec ready signal consisting of a dim white light was followed by a 1-sec foreperiod interval. The response signal was terminated when the subject pressed a response button. The intertrial interval was set at 1 sec. The appropriate RT signal intensities and frequencies (based upon the subject's equal-loudness judgments) were selected prior to each RT session. The timing and switching of events were controlled by a tape reader, a system of shielded relays, and appropriate solid-state logic. Reaction time was recorded in milliseconds by a digital printout counter.

\section{Procedure}

Initially, the subject's absolute threshold for a 1,000- $\mathrm{Hz}$ tone was determined, using the method of limits. Subsequently, equalloudness matches were conducted over four daily experimental sessions, one for each of the 20-, 40-, 60-, and 80-phon intensity levels. During each session, matches for each of the stimulus frequencies were obtained in the following order: $5,000,100$, 10,000 , and $500 \mathrm{~Hz}$. After this procedure, response latencies were measured in each of the 20 intensity-frequency combinations that were obtained in the equal-loudness matching (including the $1,000-\mathrm{Hz}$ standard). One RT session was devoted to each of the 20 stimulus conditions, and the sessions were presented randomly. The exact intensity of each RT signal was determined by the subject's equal-loudness judgments. The method of signal presentation followed a Donders Type C RT procedure; that is, the subject responded as quickly as possible upon detection of the RT signal (a "go" trial) and withheld a response when no signal appeared (a "catch" trial). The RT sessions were divided into blocks of trials, 32 trials per block, and each block could be initiated by the subject from within the sound chamber. In order to prevent the subject from initiating a response before the RT signal was presented, the order of presentation of the $24 \mathrm{go}$ trials and 8 catch trials was randomized within each block. A total of 288 RT measures, in 12 blocks, were obtained in each of the stimulus conditions. If the subject committed an error by responding on a catch trial, that block of trials was terminated and he proceeded to the next programmed block. The incidence of errors was virtually nonexistent; that is, it was less than $1 \%$ (see also Kohfeld et al., 1981).

After measuring the response latencies to each of the $\mathbf{2 0}$ tones, 12 more blocks of trials were obtained in response to a $1,000-\mathrm{Hz}$ signal at $110 \mathrm{~dB}$, SPL. The distribution of these RT measures, $T_{R}$, served as the model of the subject's response (residual) density, $t_{r}$, that was later deconvolved from each of the RT distributions obtained in the 20 stimulus conditions.

\section{RESULTS}

Table 1 presents the results of the equal-loudness matches. Each entry represents the mean value (in $\mathrm{dB}$, 
Table 1

Means and Standard Deviations of the Subject's Equal-Loudness Judgments (in Decibels, SPL)

\begin{tabular}{|c|c|c|c|c|c|c|c|c|}
\hline \multirow{3}{*}{$\begin{array}{l}\text { Stimulus } \\
\text { Frequency } \\
\text { (in Hertz) }\end{array}$} & \multicolumn{8}{|c|}{ Stimulus Intensity (in Phons) } \\
\hline & \multicolumn{2}{|c|}{20} & \multicolumn{2}{|c|}{40} & \multicolumn{2}{|c|}{60} & \multicolumn{2}{|c|}{80} \\
\hline & Mean & SD & Mean & SD & Mean & SD & Mean & SD \\
\hline 100 & 79.70 & 1.06 & 89.60 & .97 & 92.70 & 1.34 & 95.30 & .67 \\
\hline 500 & 36.90 & .99 & 43.30 & 1.25 & 62.80 & 1.93 & 78.70 & .95 \\
\hline 5,000 & 40.80 & 1.40 & 49.70 & 1.25 & 64.10 & .74 & 80.30 & 1.77 \\
\hline 10,000 & 49.00 & $\begin{array}{l}1.40 \\
1.76\end{array}$ & 59.50 & 1.84 & 72.30 & 1.06 & 84.00 & 1.25 \\
\hline
\end{tabular}

Note $-1,000 \mathrm{~Hz}$ was the standard frequency for the equal-loudness matches.

SPL) of 10 loudness matches between the $1,000-\mathrm{Hz}$ standard and the various comparison tones. From Table 1, it is evident that (1) much greater levels of signal energy were required at the high and low frequencies for loudness to remain constant, (2) the effects of frequency became less pronounced as stimulus intensity increased, and (3) the subject produced very consistent equal-loudness judgments, as indicated by the low standard deviations. An extended discussion of the effects of stimulus intensity and frequency on judgments of equal loudness is provided elsewhere (Kohfeld et al., 1981).

After the tones that differed in frequency were judged to be equally loud, they were presented as RT signals at specific intensities, depending on the particular values obtained from the equal-loudness matches. The observed RT distributions that resulted are shown in Figure 1, as denoted by the solid lines. Each distribution is based on $\mathbf{2 8 8}$ observations, and the bin size is $5 \mathrm{msec}$. The ordinate of each distribution indicates the proportion of responses falling into each of the successive 5-msec intervals. Also indicated are the mean and standard deviation of each distribution. The densities represented by the dashed lines are described subsequently in the section of this paper called "Reconvolutions."

Reading down a column in Figure 1 provides a comparison across intensities at a particular frequency, while reading across a row allows a comparison across frequencies at a given intensity level. The former comparison reveals that RT is inversely related to signal intensity, as indicated by the progressive increase in mean RT and the corresponding shift of the observed RT distributions along the time axis. Moreover, if equal loudness were to generate equal RT, then a comparison at each intensity across the five frequencies should yield similarly shaped distributions (e.g., the means and standard deviations should be about the same). Although the equal-loudness, equalRT relationship was upheld at 80 and 60 phons, equally-loud tones did not yield equivalent RTs at 40 and 20 phons across the signal frequencies. Specifically, as found in two previous investigations (Kohfeld et al., 1981; Santee \& Kohfeld, 1977), RTs at 40 and 20 phons to $1,000-\mathrm{Hz}$ signals were longer than those to the other frequency values at these in- tensities. Moreover, the overall shapes of the RT distributions appeared to differ across frequencies, especially at 20 phons.

Figure 2 presents a smoothed version of $T_{R}$, or the RT distribution obtained in response to the $1,000-\mathrm{Hz}$ tone at 110 phons, along with its mean of $136 \mathrm{msec}$ and standard deviation of $9.1 \mathrm{msec}$. The distribution is based on 288 RT measures, and the bin size is $5 \mathrm{msec}$. This response distribution, $T_{R}$, was smoothed once by a method of running averages known as the "hanning" technique. ${ }^{1} \mathrm{We}$ think that smoothing $T_{R}$, which later served as the model of $t_{r}$, resulted in a more reliable application of the deconvolution process without sacrificing too much detail in the original response distribution.

\section{DECONVOLVING THE REACTION-TIME DISTRIBUTIONS}

\section{Background Considerations}

The main assumption which underlies our analyses is that the overall RT distribution is a convolution of two independent, component densities representing the detection stage, $t_{d}$, and the response (or residual) stage, $t_{r}$. As shown in Figure 2, we obtained an empirical estimate of $t_{r}$, called $T_{R}$, by generating its distribution from RTs to a very strong signal in which the $t_{d}$ component was assumed to be negligible. Our next task was to develop analytic methods which were suited to the identification of $t_{d}$.

The point of departure was to consider the basic equation

$$
\mathbf{x}=\mathbf{y}+\mathbf{z}
$$

where $x, y$, and $z$ are continuous random variables with probability density functions $v, h$, and $u$, respectively. It is well known (see, e.g., Hoel, Port, \& Stone, 1971) that if $y$ and $z$ are statistically independent, then

$$
v(x)=\int_{-\infty}^{\infty} h(y) u(x-y) d y .
$$

Equation 2 is called a convolution integral, and therefore the process of finding $h$, given $u$ and $v$, is some- 

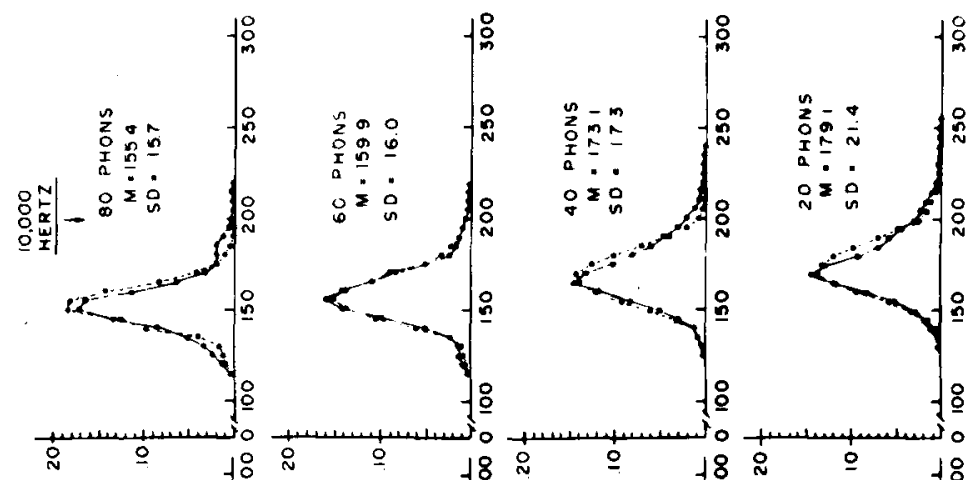

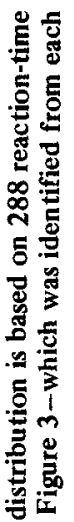
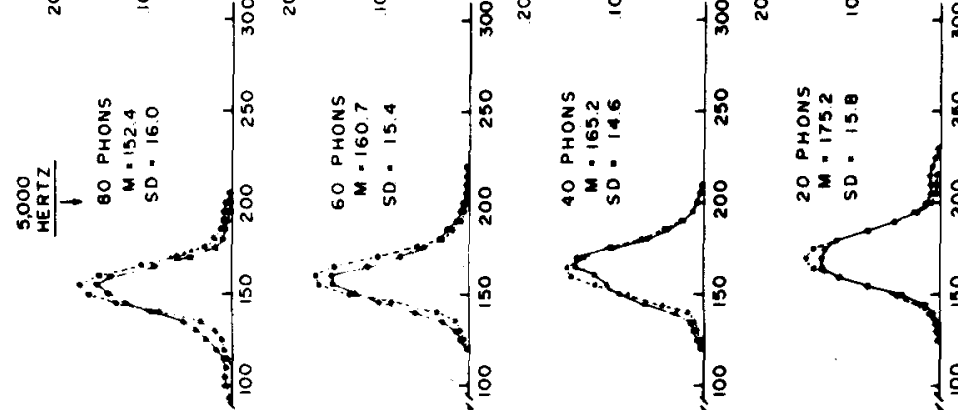

है
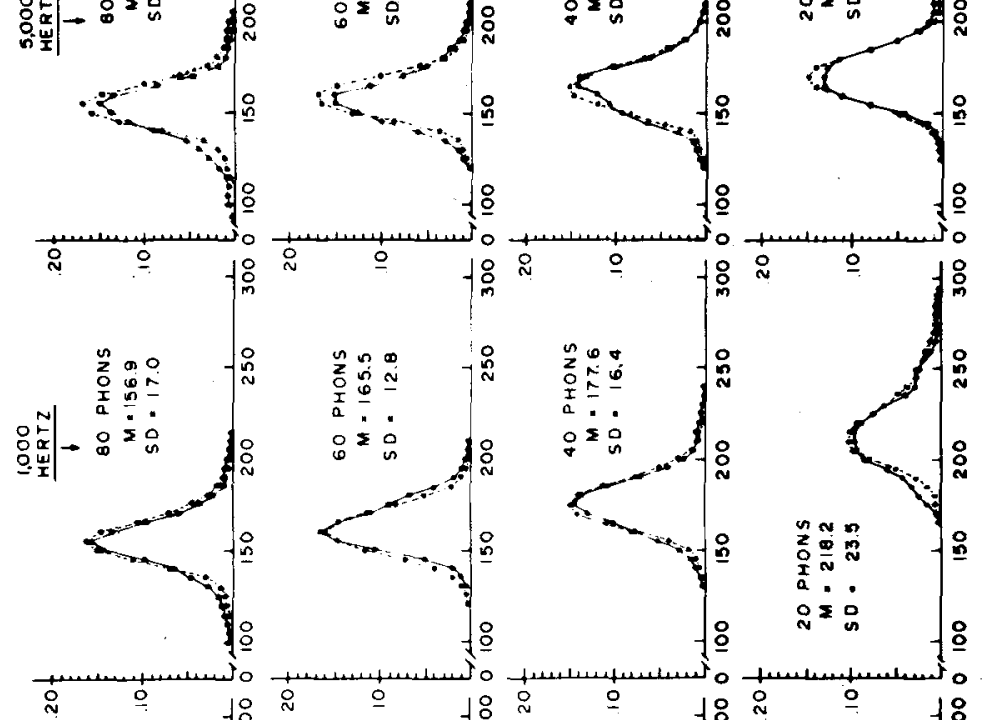

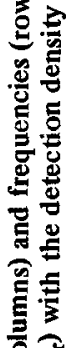
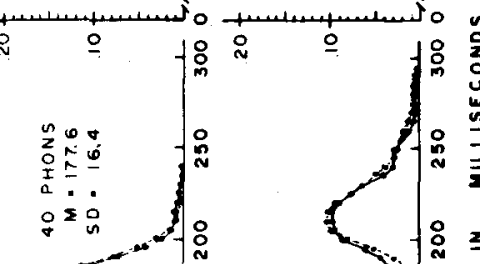

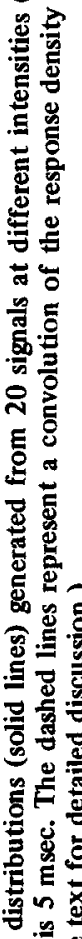
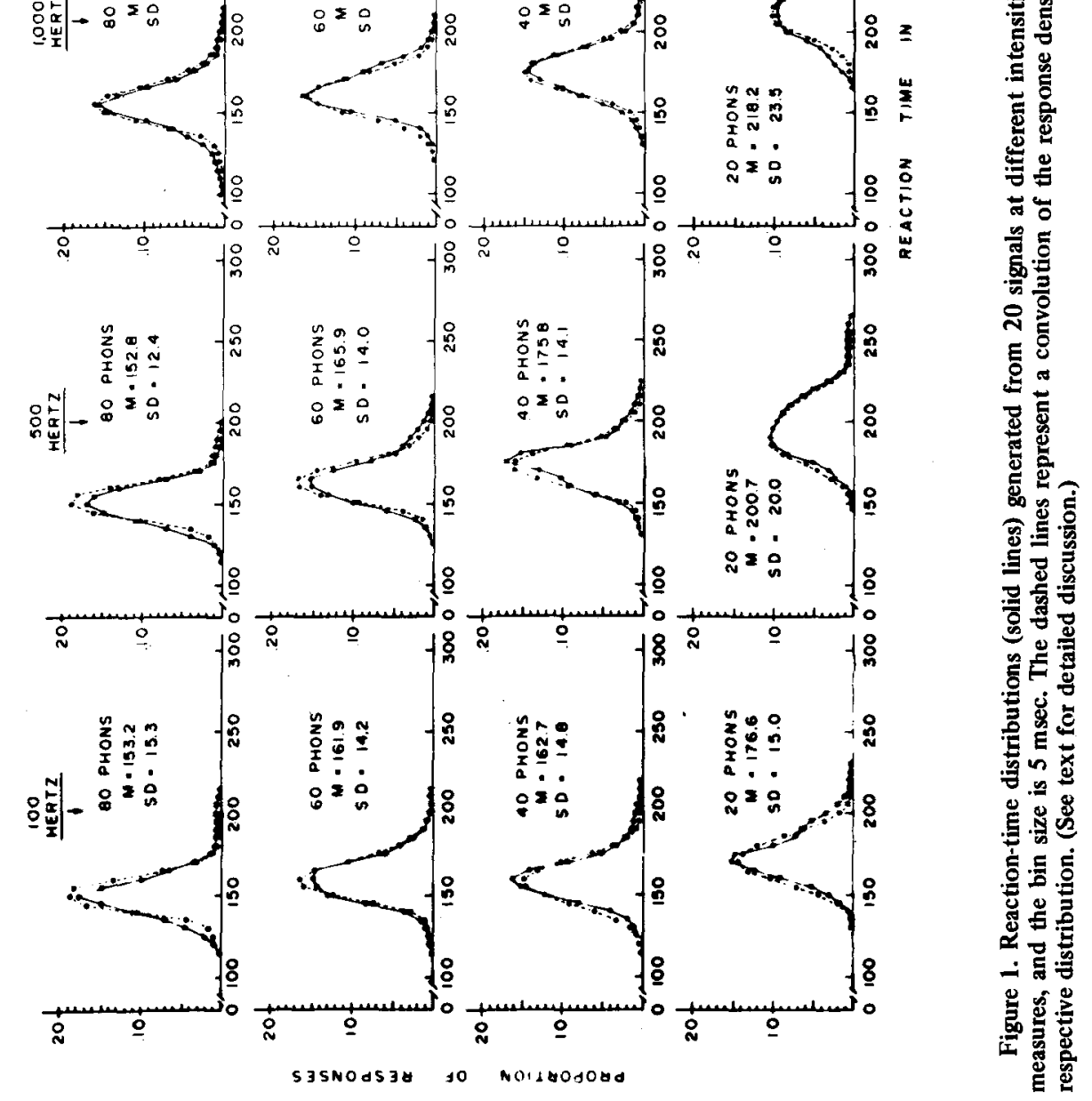


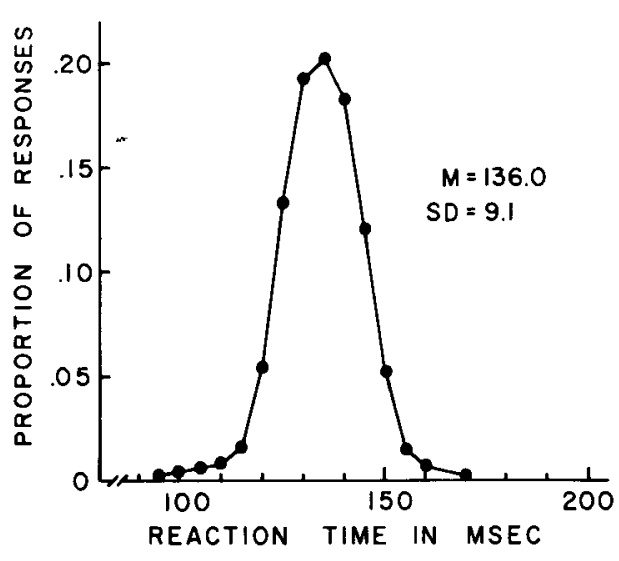

Figure 2. Reaction-time distribution generated from a 110-phon, 1,000-Hz signal. The distribution contains 288 reaction-time measures, and the bins are 5 msec wide. This distribution served as the response density, $t_{r}$, which was deconvolved from each of the 20 distributions shown in Figure 1.

times called "deconvolution." We will usually call this process "identification," a term which is more common in the vocabulary of linear systems identification (Hsia, 1977; Sage \& Melsea, 1971).

The Fourier transform of Equation 2 is given by

$$
\mathrm{V}(\omega)=\mathrm{H}(\omega) \mathrm{U}(\omega),
$$

where the capital letters $\mathrm{V}, \mathrm{H}$, and $\mathrm{U}$ are the Fourier transforms of $v, h$, and $u$, respectively. It is known that if one has the Fourier transforms of $u$ and $v$, the transform of $h$ can be obtained, and the inverse transform yields the identification of $h$. Since this solution is now quite practical to derive (Green \& Luce, 1971), we utilized a fast Fourier transform (FFT) program in order to compute transforms of RT and $T_{R}$, and thus obtain the transform of the desired component, $t_{d}$. What resulted was a chaotic estimate of $t_{d}$, which was similar to the initially poor results obtained by Green and Luce (see their Figure 5, p. 261; we also obtained extreme oscillations and negative values in the estimated densities of $t_{d}$ ).

It could be suggested that our difficulty with the Fourier transform arose from the relatively small number of $R T$ measures $(N=288)$ in each of our distributions. There are at least two reasons why we do not think that sample sizes were the primary source of our problems with Fourier analysis. First, the data were taken from a well-trained subject who had more than a year of prior experience with the same Donders $C$ paradigm. Based on block-by-block feedback of both his RT means and standard deviations, our subject achieved remarkably consistent RT performance across trial blocks, as evidenced by the generally small standard deviations reported in Figure 1. Moreover, his RT data are not characterized by marked irregularities in the frequency distributions. Second, we had previously attempted to apply Fourier analysis to RT distributions comprising 1,440 measures, taken from the same highly practiced subject in another experiment. Even with these larger sample sizes of similarly consistent RT measures, we were unsuccessful with the Fourier transform.

Perhaps a basic reason for the initial failures of Fourier analysis of RT distributions is that $v$ and $u$, in Equation 2, are not generated as continuous functions, but are empirically obtained samples at discrete intervals (i.e., they are histograms with prescribed bin widths in milliseconds). Thus, Equation 2 can appropriately be written as

$$
v_{k}=\sum_{i=0}^{k} h_{i} u_{k-i},
$$

where the subscripts indicate the number of the sample at some implied sampling interval $\Delta$, the bin width; for example, $v_{k}$ is $v(k \Delta)$. One could still take the discrete Fourier transform of Equation 4 and solve for the samples of $h$. However, since the record length from which the estimates of $u$ and $v$ are obtained is finite, numerical instability occurs when computing the required Fourier transforms. As noted by Green and Luce, some of the numerical problems can be alleviated by heavily smoothing the estimates of $u_{k}$ and $v_{k}$, but at the risk of destroying vital information about the actual forms of the obtained distributions. In this vein, we tried Fourier analysis after smoothing $R T$ and $T_{R}$ three times, using the hanning technique. Even then, highly unstable and unrealistic estimates of $t_{d}$ were obtained. Because of the problems associated with Fourier analysis of RT data, we decided to evaluate another approach to the identification of $t_{d}$.

\section{System Identification of Detection Models}

System identification is concerned with a technique which enables us to determine a mathematical model for a process whose input-output relationships are already known (Hsia, 1977). In terms of our present analysis, a systems-identification approach implies that the observed RT is the output of a linear system comprising a known input process $\left(T_{R}\right)$ and another, hypothetical, input process $\left(t_{d}\right)$ for which we seek to identify a model. What follows is a description of how we applied linear systems identification to our RT data.

Linear difference equations. Consider the Z-transform (Jury, 1964) of a discrete function, $f$ :

$$
F(Z)=\sum_{k=0}^{\infty} f_{k} Z^{-k}
$$

Equation 5 may be regarded as a generating function for the samples $f_{k}$. According to the definition of the $\mathrm{Z}$ transform, as shown by Jury (1964), it can be shown that the $Z$ transform of Equation 4 is 


$$
\mathrm{V}(\mathrm{Z})=\mathbf{H}(\mathrm{Z}) \mathrm{U}(\mathrm{Z})
$$

It may be seen that there is a direct relationship between Equation 2 and Equation 3 for the continuous case, and between Equation 4 and Equation 6 for the discrete case.

We may now draw an analogy between the problem at hand and the identification problem in linear systems identification (Jenkins \& Watts, 1969). Equation 2 can be visualized as the result of passing $u$ through a linear system with impulse response $h$. It is well known that the output of a continuous linear system can be characterized by a linear differential equation. In the discrete case, Equation 4 can be visualized as the result of passing samples of $u$ through a discrete linear system with an impulse response given by the samples of $h$. The output of this discrete linear system can be characterized by the linear difference equation

$$
\begin{aligned}
& v_{k}-q_{1} v_{k-1}-q_{2} v_{k-2}-\ldots-q_{m} v_{k-m} \\
& \quad=b_{0} u_{k}+b_{1} u_{k-1}+b_{2} u_{k-2}+\ldots b_{n} u_{k-n} .
\end{aligned}
$$

By repeated use of the shift theorem for $Z$ transforms (Jury, 1964), the $Z$ transform of Equation 7 is

$$
\begin{gathered}
V(Z)-q_{1} Z^{-1} V(Z)-q_{2} Z^{-2} V(Z)-\ldots-q_{m} Z^{-m} V(Z) \\
=b_{0} U(Z)+b_{1} Z^{-1} U(Z)+\ldots+b_{n} Z^{-n} U(Z)
\end{gathered}
$$

Accordingly,

$$
\begin{aligned}
V(Z) & \left(1-q_{1} Z^{-1}-q_{2} Z^{-2}-\ldots-q_{m} Z^{-m}\right) \\
= & U(Z)\left(b_{0}+b_{1} Z^{-1}+b_{2} Z^{-2}+\ldots+b_{n} Z^{-n}\right),
\end{aligned}
$$

or

$$
V(Z)=\frac{\left(b_{0}+b_{1} Z^{-1}+b_{2} Z^{-2}+\ldots+b_{n} Z^{-n}\right)}{\left(1-q_{1} Z^{-1}-q_{2} Z^{-2}-\ldots-q_{m} Z^{-m}\right)} U(Z) .
$$

Since $v_{k}$ is the output and $u_{k}$ is the input in the application of a linear system, the polynomial in $\mathrm{Z}$ in Equation 10 is the transfer function of the system that was identified as $\mathrm{H}(\mathrm{Z})$ in Equation 6. It follows that

$$
\begin{aligned}
H(Z) & =\sum_{i=0}^{\infty} h_{i} Z^{-i} \\
& =\frac{\left(b_{0}+b_{1} Z^{-1}+b_{2} Z^{-2}+\ldots+b_{n} Z^{-n}\right)}{\left(1-q_{1} Z^{-1}-q_{2} Z^{-2}-\ldots-q_{m} Z^{-m}\right)} .
\end{aligned}
$$

In Equation 11, if the values of $b_{i}(i=0, \ldots, n)$ and $q_{i}(i=1, \ldots, m)$ are known, then the values of $h_{i}$ $(i=1,2, \ldots)$ can be computed. Thus, the problem of finding $h$ has been reduced to obtaining the parameters in the difference equation given by Equation 7. This is the reason why this analytic method is sometimes called the "parametric" approach.

Estimating the parameters. There are recursive routines which have been developed by control systems analysts for estimating the parameters in Equation 11 (Hsia, 1977; Sage \& Melsea, 1971). However, we used a procedure which is likely to be more acceptable to those who are interested in the analysis of, for example, RT distributions.

Our general approach can be described as a threestep process: (1) Choose reasonable numbers for $\mathrm{m}$ and $\mathrm{n}$ in Equation 7, which relates the output values to the input values; (2) compute a stepwise, multipleregression analysis to determine the values of $q_{i}$ and $b_{i}$ which yield the best, least squares fit for Equation 7; and (3) from knowledge of the values $q_{i}$ and $b_{i}$, use Equation 11 to determine the values of $h_{\mathrm{i}}$.

Initially, we decided to execute Step 1 by choosing a reasonably large number for both $m$ and $n$ (e.g., 6 or 8), and to let the stepwise multiple-regression routine do the work of eliminating those variables that were not significant in the regression at a chosen level of significance. It became obvious that this "best of all possible models" approach had limitations, mainly because of the variability that is inherent in all RT data. Accordingly, it was to our advantage to have some prior knowledge about the probability density to be identified in order to help us select reasonable values for $m$ and $n$. For example, it was possible for us to estimate the mean value of $t_{d}$, since $t_{d}=R T-T_{R}$, where $R T$ and $T_{R}$ had known values. As discussed subsequently, this information about known mean values proved useful in the selection of the most appropriate models for the entire shapes of $t_{d}$.

The computations in Step 2 are straightforward because of the availability of a stepwise, multipleregression routine in the most recent version of SPSS. In our early applications of Step 2, we allowed several nonzero values of $b_{i}$ in the regression analyses. After deconvolving a wide variety of unimodal distributions, it became obvious that the use of more than one significant $b_{i}$ in the regression analysis did not provide additional useful information about the shapes of the component distributions that were to be identified. Consequently, the outcomes of the regression analyses reported in our research involved only one nonzero value of $b_{i}$. While it is clear that more than one significant $b_{i}$ in Equation 7 would obtain if it were suspected that the unknown distributions were multimodal, this simply does not follow because of the unimodal shapes of our obtained RT distributions. Thus, by using only one $b_{i}$, we have limited our general class of models to unimodal dis- 
tributions, a restriction which seems reasonable for at least two reasons: (1) All of the empirical RT distributions (the $T_{R}$ input and the $R T$ outputs) from which our models were derived are unimodal; and (2) each of our component models, when reconvolved on the input distribution, $T_{R}$, provided a very satisfactory estimation of the particular output distribution, RT, from which the model was originally identified (see the dashed lines in Figure 1 and appropriate discussion). It appears that Box and Jenkins' (1976) discussion of the principle of parsimony in the construction of mathematical models describes our procedure rather well. In particular, they note that it is "important to employ the smallest possible number of parameters for adequate representation" (p. 17). Considering the experimental variation inherent in RT data, if we were to employ, say, six values of $b_{i}$ when one would suffice, we might needlessly obtain a somewhat more complex model when a more simple and adequate one would suffice.

Finally, Step 3 could be performed by the process of long division. However, a more expedient method is to multiply both sides of Equation 11 by the denominator of $H(Z)$, and to equate coefficients of $Z^{-i}$ on both sides of the equation. When this was done, the following, recursive relation was derived:

$$
h_{i}=b_{i}+\sum_{j=1}^{\min (i, m)} h_{m-j} q_{j} .
$$

Equation 12 can be readily programmed for solution by a computer. In fact, we have developed a program which not only computes $h_{i}$, but also determines the mean value for the desired distribution we seek to identify.

In view of the foregoing considerations, we modified our original, three-step approach for estimating the parameters of the densities we sought to identify, as follows: (1) In Equation 7, choose m equal to some relatively large number (we used $m=6$ in our analyses), and subsequently test individual values of $u_{k-i}$, as determined by the significance of each in the regression analysis; (2) compute a stepwise, multipleregression analysis to determine the coefficients of the significant variables in Equation 7 for each choice in Step 1; and (3) determine the values of $h_{i}$ for the best result obtained in Step 2. While the word "best" implies a rather subjective interpretation, we followed three specific guidelines in our completion of Step 3: (1) a high coefficient of correlation (.96 or higher) for the specific choice selected in Step 2; (2) a mean value which approximates most closely the known mean value for the density to be identified; and (3) a convolution of the derived, best models of $t_{d}$ (usually one or two) with $T_{R}$ which most closely approximates the original, composite $\mathrm{RT}$ distribution. This last criterion was the most important test of the final version of each $t_{d}$ that was accepted.

\section{THEORETICAL ANALYSIS OF THE COMPONENT DENSITIES}

\section{Detection Densities}

An important assumption in our analysis was that the response component, $t_{r}$, remained constant at all levels of intensity and frequency employed in our design. This is because the same Donders $C$ paradigm was used throughout experimentation; that is, the foreperiod interval was $1 \mathrm{sec}$, and the ratio of "go" trials to "catch" trials was always 3:1. Furthermore, the same, highly practiced subject was used in all of the conditions, including $T_{R}$. When the variables of expectancy and set are controlled in this manner (particularly the constant foreperiod duration), Hohle (1965) has also argued for a relatively stable response component. Assuming that $t_{r}$ does remain the same, the variations in the observed RT distributions, as seen in Figure 1, should reflect corresponding changes in $t_{d}$ that result from fluctuations due to signal intensity and frequency.

The process of identification (deconvolution) followed the procedures outlined previously, thus yielding the most appropriate density for the particular detection process which underlies each of the observed RT distributions shown in Figure 1. These 20 models of $t_{d}$ are shown in Figure 3. A view down each column permits a comparison across intensities, while a view across each row allows one to compare the models of $t_{d}$ across frequencies. The ordinate of each detection density indicates the proportion of times a "detection response" occurred within each of the 5-msec bins.

It is evident from Figure 3 that detection at $\mathbf{8 0}$ and 60 phons is characterized by a family of exponential densities, whereas detection at 40 and 20 phons appears to range from gamma to near normal in form. The two exceptions are the 60-phon, $1,000-\mathrm{Hz}$ density which looks either gamma or normal, and the somewhat irregular model at 40 phons, $500 \mathrm{~Hz}$ which appears "borderline" between gamma and exponential. In this respect, it should be noted that the exponential can be viewed as a special case of the gamma, so clear-cut distinctions between these two densities are not always appropriate. Furthermore, a lower order gamma and a normal distribution are similar in form; we retain these distinctions simply for clarity of discussion. Since the shapes of our detection models can range from sharply exponential to normal, the selection of the appropriate model (gamma, exponential, or normal) can sometimes be arbitrary, particularly if this selection is based solely on the appearance of the densities, that is, as shown in Figure 3.

In order to provide additional rationale for the selection of appropriate models, Table 2 gives detailed information about each density shown in Figure 3. The first two columns identify the particular intensity- 


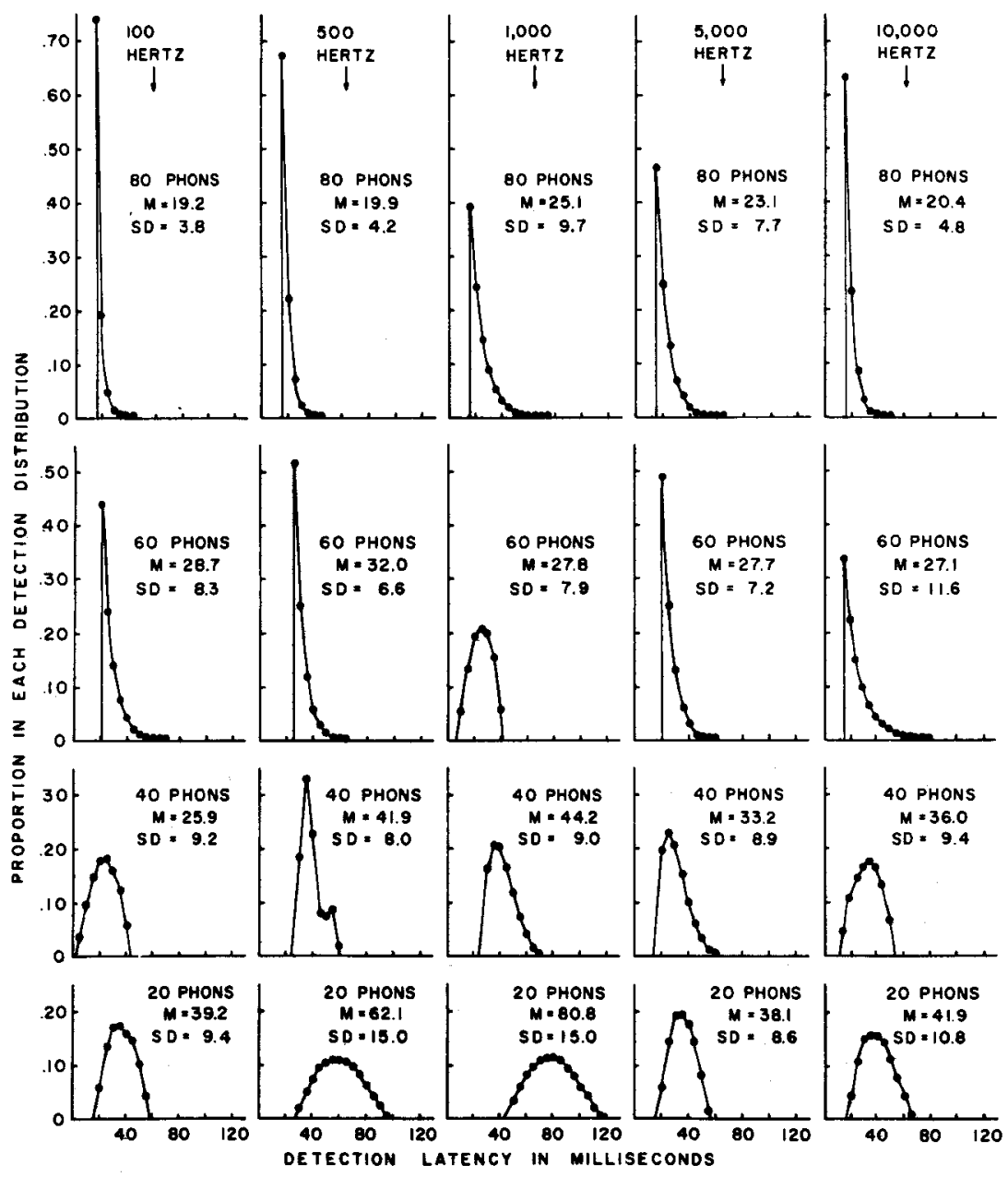

Figare 3. Sensory-detection densities ( $t$ ) obtained by deconvolving $t$, (Figare 2) from each of the corresponding reaction time distributions shown in Figure 1. The data are plotted in 5-msec bins. (The convolution of $t_{r}$ with each $t_{d}$ model above yields the corresponding density, at that particular intensity and frequency, Indicated by the dashed lines in Figure 1.)

frequency value, and the next two columns give the mean detection latency (DL) and the standard deviation of the detection latency (SD, DL), respectively; these two statistics are also shown in Figure 3. The next column indicates the time delay $(t)$ between zero and the first bin in which detection responses were observed. In the case of an exponential density, the value $t$ is the fixed time delay. The criterion column (n) lists the estimated number of events (or "impulses") that underlie each density; this estimate is based on the a priori assumption that all the densities are gamma in form. ${ }^{2}$ The relation between gamma densities and underlying "neural impulse" counts will be discussed subsequently.

Although it seems obvious from Figure 3 that about half of the densities are exponential in form, we thought the calculation of the gamma parameter $n$ would also prove useful in helping to determine whether the parent density was exponential, gamma, or perhaps normal in form. This was because an exponential is known to have one underlying event, whereas gamma and normals represent the convolution of two or more exponentials, and thus are characterized as having two or more underlying events. In this vein, we have found that the convolution of, for example, four or five exponential densities usually causes the shape of gamma to resemble the normal distribution. That is, as $\mathbf{n}$ becomes greater than two, the gamma distribution becomes less skewed and progressively more symmetrical. An excellent discussion of the transition from exponential to gamma to normal may be found in Benjamin and Cornell (1970).

Returning to Table 2, the number of impulses depicted in the criterion column was indicative of whether the various detection densities were either exponential or gamma. Thus, if the number of criterion impulses was one, the density was regarded as exponential, whereas if two or more impulses were obtained, either 
Table 2

Exponential and Gamma Models Applied to the Detection Densities Shown in Figure 3

\begin{tabular}{|c|c|c|c|c|c|}
\hline \multirow{2}{*}{$\begin{array}{l}\text { Stimulus } \\
\text { Intensity }\end{array}$} & \multirow{2}{*}{$\begin{array}{l}\text { Stimulus } \\
\text { Frequency }\end{array}$} & \multicolumn{2}{|c|}{ DL } & \multirow{2}{*}{$\begin{array}{c}\text { Delay } \\
(\mathrm{t})\end{array}$} & \multirow{2}{*}{$\begin{array}{l}\text { Criterion } \\
\text { (n) }\end{array}$} \\
\hline & & Mean & SD & & \\
\hline \multicolumn{6}{|c|}{ Gamma Distributions } \\
\hline 20 & 100 & 39.2 & 9.4 & 20 & 4 \\
\hline 20 & 500 & 62.1 & 15.0 & 30 & 5 \\
\hline 20 & 1,000 & 80.8 & 15.0 & 50 & 4 \\
\hline 20 & 5,000 & 38.1 & 8.6 & 20 & 4 \\
\hline 20 & 10,000 & 41.9 & 10.8 & 20 & 4 \\
\hline 40 & 100 & 25.9 & 9.2 & 5 & 5 \\
\hline 40 & 500 & 41.9 & 8.1 & 30 & 2 \\
\hline 40 & 1,000 & 44.3 & 9.0 & 30 & 3 \\
\hline 40 & 5,000 & 33.2 & 8.9 & 20 & 2 \\
\hline 40 & 10,000 & 36.0 & 9.4 & 15 & 5 \\
\hline \multicolumn{6}{|c|}{ Exponential Distributions } \\
\hline 60 & 100 & 28.7 & 8.3 & 20 & 1 \\
\hline 60 & 500 & 32.0 & 6.6 & 25 & 1 \\
\hline 60 & 1,000 & 27.9 & 7.9 & 10 & $5^{*}$ \\
\hline 60 & 5,000 & 27.7 & 7.2 & 20 & 1 \\
\hline 60 & 10,000 & 27.1 & 11.6 & 15 & 1 \\
\hline 80 & 100 & 19.3 & 3.8 & 15 & 1 \\
\hline 80 & 500 & 19.9 & 4.2 & 15 & 1 \\
\hline 80 & 1,000 & 25.2 & 9.7 & 15 & 1 \\
\hline 80 & 5,000 & 23.1 & 7.7 & 15 & 1 \\
\hline 80 & 10,000 & 20.4 & 4.8 & 15 & 1 \\
\hline
\end{tabular}

Note-Stimulus intensity is given in phons; stimulus frequency is given in hertz. Detection latency (DL) and delay are given in milliseconds. "The 60-phon distribution at 1,000 Hz is gamma.

a higher order (near exponential) or a lower order (near normal) gamma was indicated. This information is listed in the last column of Table 2. If one compares the kind of distribution selected in Table 2 with the actual forms of these densities that are shown in Figure 3, no obvious discrepancy exists between these two sources of information about the shapes of our detection densities. ${ }^{3}$

The exponential models of $t_{d}$ imply that the detection process operates much like a constant, conditional probability mechanism. A lower bound of an exponential distribution represents, in this case, a fixed time delay from signal onset to when the detector receives enough neural information for signal detection. The probability that detection will occur prior to the constant delay is essentially zero. An upper bound represents the point at which the decay function reaches zero. Obviously, the probability that detection will occur between the lower and upper bounds of an exponential distribution is 1.0. More importantly, it can be shown that the conditional probability of a temporal event described within the upper and lower bounds of an exponential distribution is fixed, and independent of any arbitrary time delay (see McGill, 1963). For instance, if the detection of an 80-phon, $1,000-\mathrm{Hz}$ tone (see Figure 3) has not occurred by $20 \mathrm{msec}$, then the probability that detection will occur beyond, for example, $25 \mathrm{msec}$, is equal to the probability that detection will occur beyond $30 \mathrm{msec}$, given that it has not already occurred by $25 \mathrm{msec}$. In more general terms, if the detector fails to respond to a signal up to time $t$, for any $t$ above the lower bound, this failure does not alter one's prediction of the length of time (from $t$ ) before the detector will respond. Incidentally, this description also characterizes, for example, the time between arrivals to a queue when the arrivals are characterized by a Poisson probability law.

As noted previously, the relation between exponential and gamma distributions (including gammas with normal or near-normal shapes) is straightforward; that is, a gamma random variable is simply the sum of two or more exponential random variables. One way to conceptualize the events that underlie our gamma and/or normal densities at 20 and 40 phons is to assume that signal detection did not occur in response to only one impulse, as was the case with an exponential process; instead, the detector required several impulses before it was satisfied that a signal was present. In this vein, let us assume that signal detection in the RT process involves the continuous monitoring of neural activity in the detection mechanism. The onset of a RT signal initiates an abrupt change in the level of spontaneous neural activity (or "neural noise") in the auditory sensory system. This change in the signal-to-noise ratio might be described as a series of neural events or "impulses" which is processed by the detector. If a signal is sufficiently strong (i.e., 60 or 80 phons), only one impulse is necessary to meet the detection criterion (n). This can be inferred from the fact that an exponential density $(n=1)$ best describes the detection process for strong signals (see Figure 3 and Table 2). However, for relatively weak RT signals (i.e., 20 or $\mathbf{4 0}$ phons), the detection criterion requires more impulses before it is satisfied that a signal is present. The basis for this latter deduction is the observed, gamma-like densities that characterize detection at weak intensity levels. Of course, even when the criterion count is fixed (e.g., $n=1$ ), detection times for a given $R T$ signal will vary from trial to trial. This variability is described by the value of $\lambda$, the rate of decay in an exponential density. For weaker signals, the trial-to-trial variability can be described by the value of $\lambda$ in a gamma density. Assuming gamma, $\lambda$ refers to the average rate at which impulses are accumulated in the detector, whereas $\mathrm{n}$ specifies the criterion number that must accrue before the detector will fire. ${ }^{4}$

Returning to Figure 3, it is noteworthy that all of the exponential densities at 80 phons are very sharply peaked. For example, at $100 \mathrm{~Hz}, 80$ phons, the detection process exhibits little variability, almost to the point of being a fixed time delay. If this were true, the detection density would be represented by a delta function (or "spike") at some point on the time axis, with virtually no variability. Indeed, almost all of the variability in the overall $\mathrm{RT}$ distributions at 80 phons (see Figure 1) is based on $t_{r}$ (see Figure 2), the response 
process. This supports our belief that the much more intense, 110-phon signal did, in fact, generate a RT distribution based almost entirely on $t_{r}$.

Finally, it may be observed that the shapes of the detection components differed across stimulus frequencies as well as stimulus intensities. For example, there are subtle differences in the shapes of the exponentials at 80 phons (see Figure 3 ). Since the time delay and the criterion number are the same across all five frequencies at 80 phons (see Table 2), any differences in the density shapes are attributable to variation in $\lambda$. Moreover, at 20 phons, the criterion counts (n) are nearly identical across frequencies, but the five densities have different, lower order gamma shapes. This again suggests that any effect of stimulus frequency on detection latency is reflected in $\lambda$. In other words, for stimulus intensities that were initially judged to be equivalently weak across five stimulus frequencies, the onset of these stimuli, when used as RT signals, generated different impulse rates (gamma $\lambda$ ) at these five different frequencies. However, at equivalently strong stimulus intensities, the frequency (in $\mathrm{Hz}$ ) of the RT signal influenced the temporal precision with which a single impulse is detected (exponential $\lambda$ ).

\section{Response Density}

The distribution of $T_{R}$ presented in Figure 2 resembles a Laplace distribution. McGill (1963) has described a Laplace distribution as being "essentially two exponential distributions turned back to back, resulting in a density function that is more sharply peaked than a normal variable" (p. 335). Kristofferson (1976) has also obtained similar highly peaked, symmetrical RT distributions from a practiced subject (see his Figure 9, p. 94). A Laplace distribution suggests the operation of a highly periodic latency mechanism that is well timed, practically invariant, and probably not based on a random, underlying variable. Furthermore, the Laplace often describes a mechanism in which excitations are input at regular intervals, but responses to the input can be subject to minor delays. This description is consistent with our general view of simple reaction time; once the detection mechanism is "satisfied" that a signal has occurred, its output directs the response mechanism to execute. Delays in responding to this output may result from minor fluctuations in the subject's attention or readiness to respond from trial to trial. Whatever the source of variability in $t_{r}$ may be, it is clear from its Laplace form that the delays between excitation and response are minimal.

\section{Reconvolutions}

The most important test of the adequacy of our detection components is the reconvolution of each of the estimated densities in Figure 3 with the response distribution shown in Figure 2. Thus, each distribu- tion of $t_{d}$ in Figure 3, when reconvolved with $t_{r}$, should approximate the corresponding, composite probability distribution from which it was originally identified. In Figure 1, the distributions comprising the dashed lines represent the distributions obtained by means of reconvolution. While it appears obvious that the reconvolutions (predicted densities) closely approximate the observed RT distributions, we applied the chi-square tests for goodness of fit to each of the 20 pairs of distributions shown in Figure 1. In each case, the null hypothesis was that the predicted (reconvolutions) and the obtained distributions were the same. We decided to adopt the .05 level of significance. The number of degrees of freedom for each chi-square test was determined by (1) the number of RT bins, or samples, in which comparisons were made, minus (2) one, for the estimate of $u_{k}$, minus (3) the number of estimates of $v_{k}$ that were utilized, minus (4) one, for the constraining number of RT measures in each distribution $(\mathrm{N}=\mathbf{2 8 8})$. The number of estimates of $v_{k}$ varied from a minimum of one (e.g., at $100 \mathrm{~Hz}$, 80 phons) to a maximum of three (e.g., at $500 \mathrm{~Hz}$, 20 phons).

Table 3 shows the obtained chi-square values, the appropriate degrees of freedom, and the $p$ values for the 20 chi-square tests. In all cases, the chi-square values were not large enough to reject the hypothesis at the .05 level. For example, the comparison at 20 phons, $100 \mathrm{~Hz}$ involves $16 \mathrm{df}$, for which a chi-square value equal to or greater than $\mathbf{2 6 . 2 9 6}$ is necessary to reject the null hypothesis that the predicted and observed distributions are the same. In Table 3, the column marked " $p>$ " indicates the $p$ value from

Table 3

Chi-Square Goodness-of-Fit Tests Applied to the 20 Pairs of Distributions Shown in Figure 1

\begin{tabular}{crrrr}
\hline $\begin{array}{c}\text { Stimulus } \\
\text { Intensity }\end{array}$ & $\begin{array}{c}\text { Stimulus } \\
\text { Frequency }\end{array}$ & \multicolumn{1}{c}{$\mathrm{x}^{2}$} & $\mathrm{df}$ & $\mathrm{p}>$ \\
\hline 20 & 100 & 19.87 & 16 & .30 \\
20 & 500 & 30.20 & 21 & .05 \\
20 & 1,000 & 15.42 & 25 & .90 \\
20 & 5,000 & 16.09 & 18 & .50 \\
20 & 10,000 & 20.06 & 22 & .50 \\
40 & 100 & 19.00 & 17 & .30 \\
40 & 500 & 14.13 & 16 & .50 \\
40 & 1,000 & 11.38 & 18 & .80 \\
40 & 5,000 & 13.23 & 14 & .50 \\
40 & 10,000 & 23.52 & 19 & .20 \\
60 & 100 & 5.13 & 17 & .99 \\
60 & 500 & 17.48 & 15 & .20 \\
60 & 1,000 & 20.25 & 15 & .10 \\
60 & 5,000 & 21.45 & 17 & .20 \\
60 & 10,000 & 6.92 & 19 & .99 \\
80 & 100 & 25.79 & 18 & .10 \\
80 & 500 & 13.68 & 14 & .30 \\
80 & 1,000 & 18.14 & 21 & .50 \\
80 & 5,000 & 25.71 & 19 & .10 \\
80 & 10,000 & 23.53 & 18 & .10 \\
\hline
\end{tabular}

Note-Stimulus intensity is given in phons; stimulus frequency is given in hertz. 
the table of chi-square which the corresponding obtained chi-square value did not quite reach. For example, at 20 phons, $500 \mathrm{~Hz}$, a $\chi^{2}(21)=30.20$ has a $\mathrm{p}$ value greater than .05 but less than .10; this particular comparison came closest to rejecting the hypothesis that the observed and predicted distributions were the same. In general, we think that the chi-square tests were a rather conservative (sensitive) test of the goodness of fits between the predicted and obtained distributions in Figure 1. Consequently, the hypothesis that the reconvolutions were good estimates of the observed distributions is clearly acceptable. Moreover, the success of the convolutions provides strong support for our view that the simple RT process is the composite of a uniform response density $\left(t_{r}\right)$ and a unique detection density $\left(t_{d}\right)$.

\section{GENERAL REMARKS}

One important assumption in this paper is that the response component in simple reaction time "holds still" when the same, highly trained subject is employed in a fixed RT paradigm. That is, for a given subject, the residual density $\left(t_{r}\right)$ retains its shape, but the form of the detection density $\left(t_{d}\right)$ is labile. In order to challenge this assumption, let us argue for the moment that $t_{r}$ does change, particularly as a function of stimulus intensity. If so, perhaps our models of $t_{d}$ at 80 and 60 phons are reasonably accurate representations of the detection process, but the models of $t_{d}$ at 40 phons, and especially at 20 phons, are convolutions of the "true" $t_{d}$ with some unknown component. Such an "unknown component" could be represented by the difference between the shape of $t_{r}$ at 110 phons and the shape of $t_{r}$ at, say, 20 phons. If this were the case, the family of $t_{d}$ models obtained in the present study would be rather tentative, at best.

There are at least three reasons why we believe that $t_{r}$ did, in fact, remain approximately the same across all of the stimulus conditions. First, as noted earlier, the same Donders C RT task was used in each condition. Second, the same subject was used throughout, and the variables of practice and incentive were carefully controlled. Third, and perhaps most important, it is generally accepted that changes in the response component in a RT paradigm result from variations in the foreperiod interval (e.g., Hohle, 1965; Sanders, 1977) and not from manipulations of the RT signal itself, that is, its intensity and frequency. In view of the fact that a fixed foreperiod of $1 \mathrm{sec}$ was used throughout our research, we believe that the changes in the observed RT distributions across stimulus intensity and frequency reflect changes in the detection component, $\mathrm{t}_{\mathrm{d}}$.

Despite our confidence in the stability of $t_{r}$, the design of the present study does not permit us to reach decisive conclusions about this matter. In particular, our current procedures do not rule out the possibility that $T_{R}$, even at $110 \mathrm{~dB}$, may contain a small detection component whose shape is almost surely exponential, but whose time constant is unknown. Accordingly, a necessary problem for future research is to construct a priori models of $t_{d}$ at different intensities and frequencies, and then to deconvolve these models from the empirical RT distributions to determine whether the same $t_{r}$ density will result in each case; this is an extension of the procedure outlined by Green and Luce (1971). In this vein, a more conservative approach is to deconvolve $t_{d}$ and $t_{r}$ from RT without making any prior assumptions about the shapes of these component densities. Recent work in our laboratory indicates considerable initial success in this latter endeavor. Specifically, we have found that $t_{r}$ tends to remain constant across different $R T$ tasks involving signal detection $\left(t_{d}\right)$, stimulus discrimination $\left(t_{D}\right)$, and attention switching $\left(t_{s w}\right)$; see Kohfeld, Note 2.

As a final note, we are well aware that the successful application of linear systems identification requires that the component processes that underlie the RT distribution must operate in series. Some investigators (e.g., Vorberg, Note 3) contend, based on rather compelling logic, that selection of a serial or a parallel model of the RT process should not be made at the outset, and may be virtually impossible to determine, even after careful data analysis. It is not our intent to make a decisive case for serial vs. parallel processing. However, the fact remains that linear systems identification worked satisfactorily (i.e., the reconvolutions shown in Figure 1 are quite acceptable), which provides evidence for our belief that the component stages, $t_{d}$ and $t_{r}$, operated successively. What is less clear, strictly from a linear systems point of view, is the order in which the two stages occur. However, it seems intuitively obvious that a signal must be detected before a "true" response can be initiated in the RT paradigm. Future work in our laboratory, in which multiple stages in the RT process are to be identified within the framework of linear systems, should provide additional evidence pertaining to the consecutive nature of RT components.

\section{SUMMARY AND CONCLUSIONS}

In 1963, McGill proposed that the shape of the reaction-time distribution would provide a kind of "signature" that reflected the nature of the underlying events that comprised this distribution. Since then, Green and Luce (1971) have demonstrated that simple auditory RT is the convolution of two main components: sensory detection $\left(t_{d}\right)$ and response execution $\left(t_{r}\right)$. Based on these considerations, the main goal of the present research was first to identify the components that comprise the overall RT distribution, and 
subsequently to infer the kind of hypothetical, "neural" events that give rise to the particular shapes of these components.

In order to deconvolve the overall RT distribution into its component stages, we obtained reaction times from a highly practiced subject to equally loud signals (across five frequencies) at 20-, 40-, 60-, and 80 phon intensity levels. These 20 RT distributions were treated as "outputs" within the framework of a systems-identification analysis of the RT process. The empirical "input" density was obtained by generating a RT distribution from responses to a very intense, 110-phon signal. Based on its sheer intensity, this distribution of responses was regarded as equivalent to the residual, or response, process $\left(t_{r}\right)$, free of any sensory-detection latency. When $t_{r}$ was deconvolved from each of the $20 \mathrm{RT}$ distributions, using the technique known as linear systems identification, we solved for the other "input variable"; this latter variable is best described as a family of detection densities $\left(t_{d}\right)$ that usually varies in accordance with the intensity and frequency of the RT signal. The reconvolution of each $t_{d}$ density with the uniform $t_{r}$ density provided very satisfactory approximations of the original RT distributions from which these two components were identified.

Our data indicate that the sensory-detection process at weak intensity levels is different from detection at relatively strong intensity levels. Specifically, the detection densities $\left(t_{d}\right)$ at 20 and $\mathbf{4 0}$ phons (weak intensities) reveal a detection process that generates distributions ranging from gamma to normal, whereas the detection of RT signals at 60 and 80 phons (strong intensities) yields exponential distributions. Our theory is that as few as one "neural" impulse is required for the detection of a strong RT signal, whereas two or more impulses must accumulate before a relatively weak RT signal is detected. These data are consistent with Luce and Green's (1974) general view that the detection of weak signals (in the RT paradigm) may require a psychophysical process that is somewhat different from that required in the detection of strong signals.

As a final comment, we note, with some surprise, that previous evaluations of the power-function exponents for RT-intensity functions have focused exclusively on $1,000-\mathrm{Hz}$ signals. In our present research, RT data are available across five frequencies, and one might be tempted to calculate the power exponents at the different frequencies to see how the values compare with the expected value of -.3 at $1,000 \mathrm{~Hz}$ (see, e.g., Figure 4 in Kohfeld et al., 1981). However, since the intensity levels which served as the RT signals are in phons (i.e., they are matched in loudness with $1,000 \mathrm{~Hz}$ ), they do not form orderly RT-intensity functions with respect to the unique threshold (in dB, SL) for each frequency. Moreover, there are only four data points in each intensity function (excluding the $110-\mathrm{dB}$ value at $1,000 \mathrm{~Hz}$ ). We think that at least $10 \mathrm{RT}$ values, evenly spaced between $0 \mathrm{~dB}, \mathrm{SL}$, and the strongest intensity level (expressed in sound pressure units) at each frequency, should be obtained before detailed analyses of the RT-intensity functions are made. Accordingly, the next research article in our series on loudness and reaction time will deal with the following issues: (1) When mean detection times $\left(\mathrm{Mt}_{\mathrm{d}}\right)$ are plotted as a function of stimulus intensity over a wide range of sound-pressure values, will the functions converge on $M t_{d}=0$ at the strongest intensity level (specified as $T_{R}$ ), as predicted by a successful deconvolution of $t_{r}$ from the composite RT distributions at increasing intensities? (2) As we have already predicted, will the same densities of $T_{R}$ as the one at $1,000 \mathrm{~Hz}$ also be generated at different frequencies? And (3) will these frequency-specific, $t_{\mathrm{r}}$ models, when deconvolved from overall RT distributions at increasing intensity levels within specific frequencies, generate families of $t_{d}$ models which form power functions? Obviously, considerable parsimony would be achieved if similar power exponents could describe the general relation between stimulus intensity and reaction time, regardless of stimulus frequency. In any event, if the power law holds across stimulus frequencies, any similarities between the exponents for $t_{d}$ scales (from reaction time) and the exponent for loudness would contribute to our knowledge of the power-transformation process.

\section{REFERENCE NOTES}

1. Luce, R. D. Personal communication, September 17, 1980.

2. Kohfeld, D. L. Component stages of visual information processing. Senior Research Scholar Award Presentation, Southern Illinois University at Edwardsville, March 1979.

3. Vorberg, D. On the equivalence of parallel and serial models of information processing. Paper presented at the meeting of the Mathematical Psychology Group, Los Angeles, California, August 1977.

\section{REFERENCES}

Benjamin, J. R., \& Connell, C. A. Probability, statistics, and decision for civil engineers. New York: McGraw-Hill, 1970.

Box, G. E. P., \& Jenkins, G. M. Time series analysis: Forecasting and control. San Francisco: Holden-Day, 1976.

Burbeck, S. L. Change and level detectors inferred from simple reaction times. Doctoral dissertation, University of California, Irvine, 1979.

Chatfield, C. The analysis of time series: Theory and practice. New York: Wiley, 1975.

Green, D. M., \& LuCE, R. D. Detection of auditory signals presented at random times: III. Perception \& Psychophysics, 1971, 9, 257-268.

Hoel, P. G., Port, S. C., \& Stone, C. J. Introduction to probability theory. Boston: Houghton Mifflin, 1971.

HOHLE, R. H. Inferred components of reaction times as functions of foreperiod duration. Journal of Experimental Psychology, $1965,69,382-386$. 
Hsia, T. C. System identification: Least-squares methods. Lexington, Mass: Heath, 1977.

Jenkins, G. M., \& WAtTs, D. G. Spectral analysis and its applications. San Francisco: Holden-Day, 1969.

JURY, E. I. Theory and application of the Z-transform method. Harrington, N.Y: Krieger, 1964

Kohfeld, D. L., Santee, J. L., \& Wallace, N. D. Loudness and reaction time: I. Perception \& Psychophysics, 1981, 29, 535.549.

Kristofferson, A. B. Low-variance stimulus-response latencies: Deterministic internal delays? Perception \& Psychophysics, 1976, 20, 89-100.

LuCe, R. D., \& Green, D. M. A neural timing theory for response times and the psychophysics of intensity. Psychological Review, 1972, 79, 14-57.

Luce, R. D., \& Green, D. M. Detection, discrimination, and recognition. In E. C. Carterette \& M. P. Friedman (Eds.), Handbook of perception (Vol. 2). New York: Academic Press, 1974.

McGiLL, W. J. Stochastic latency mechanisms. In R. D. Luce, R. R. Bush, \& E. Galanter (Eds.), Handbook of mathematical psychology (Vol. 1). New York: Wiley, 1963.

Restle, F. Psychology of judgment and choice. New York: Wiley, 1961.

Sage, A. P., \& Melsea, J. L. System identification. New York: Academic Press, 1971.

Sanders, A. F. Structural and functional aspects of the reaction process. In S. Dornic (Ed.), Attention and performance VI. Hillsdale, N.J: Erlbaum, 1977.

SAntee, J. L., \& Kohfeld, D. L. Auditory reaction time as a function of stimulus intensity, frequency, and rise time. Bulletin of the Psychonomic Society, 1977, 10, 393-396.

\section{NOTES}

1. We used a 3-point, moving-average smoothing technique to plot the continuous curve shown in Figure 2. This method of smoothing with weights of $.25, .50$, and .25 is called "hanning," after the Austrian meteorologist Julius Von Hann (see Chatfield, 1975, pp. 142-143). We have available a FORTRAN program for rapid computation of this smoothing function.

2. If we assume that trial-to-trial variability in the impulse rate for a given stimulus intensity can be described by a Poisson probability distribution (Restle, 1961, pp. 165-170), it follows that the trial-to-trial variability in detection latency for a subject with a fixed criterion (set number of impulses) should form a gamma distribution with parameters $\lambda$ (average impulse rate) and $\mathbf{n}$ (criterion impulse count). These parameters can be calculated from the detection densities, since it is known that $n / \lambda=$ mean $R T$, and $\mathrm{n} / \lambda^{2}=\mathrm{SD}^{2}$. By squaring both sides of the former equality, we get $n^{2} / \lambda^{2}=$ mean $^{2}$, and by substitution and reducing to lowest terms, we obtain $\mathrm{n}=$ mean $^{2} / \mathrm{SD}^{2}$. In order to apply a gamma model to our detection densities, the time delay, $t$, must be subtracted from the mean detection latency (DL) for each density. Accordingly, the ratio $(\mathrm{t}-\text { mean } \mathrm{DL})^{2} / \mathrm{SD}^{2}$ yields an estimate of the criterion count for a given detection density; these values are presented in the criterion column of Table 2.

3. A conservative criterion for selection of the "best" theoretical model for each detection density $\left(t_{d}\right)$ would be to generate for example, a series of gamma distributions for each $t_{d}$ at 20 and 40 phons, and subsequently to perform chi-square goodness-of-fit tests to evaluate the null hypothesis that the theoretical gamma distributions and the observed $t_{d}$ densities have the same shapes. Another approach would be to examine the hazard functions of the $t_{d}$ densities. As suggested by Luce (Note 1), hazard functions may reveal more about the forms of distributions than the actual density functions themselves (see also, Burbeck, 1979). Our plan is to present a detailed analysis of the forms of $t_{d}$, particularly at relatively weak signal intensities, in our next paper in this series on loudness and reaction time.

4. The ratio $n / \lambda$ is the average interarrival time (IAT) between n pulses. Luce and Green $(1972,1974)$ have proposed a theory of the detection process in which the detector compares the average IAT between pulses with a criterion IAT value. This "timing" model assumes that the detector samples the impulse train to determine whether the average IAT within a fixed, brief interval is equal to or less than the criterion IAT; if so, the detector fires. We have offered a "counting" model of detection in this paper, but anticipate that other conceptualizations (e.g., a timing model) might also account for our detection densities. It is clear that both counting and timing models may be deduced from the same distributions, and thus are closely related.

(Received for publication March 26, 1980; revision accepted March 25, 1981.) 